\title{
Case Presentation of Concomitant and Contiguous Adenomatoid Odonto- genic Tumor and Focal Cemento-Ossifying Dysplasia
}

\author{
Gita Rezvani ${ }^{1}$, Mandana Donoghue, ${ }^{2, *}$, Peter A. Reichart ${ }^{3}$ and Neda Pazuhi ${ }^{4}$ \\ ${ }^{I}$ Department of Oral and Maxillofacial Pathology, Faculty of Dentistry, Shahed University, Tehran, Iran; ${ }^{2}$ OMFP Cen- \\ ter, Belgaum, India; ${ }^{3}$ University Professor emeritus, Charité, Department of Oral Medicine, Dental Radiology and Oral \\ Surgery (Prof A.M. Schmidt- Westhausen) CC3, Assmannshauser str. 4-6, 14197, Berlin, Germany; ${ }^{4}$ Department of \\ Conservative Dentistry, Faculty of Dentistry, Isfahan University of Medical Sciences, Isfahan, Iran
}

\begin{abstract}
A 24 year-old male was presented for the diagnosis of an asymptomatic bony expansion in relation to the right maxillary canine and first premolar. The unilocular radiolucent lesion with central foci of calcification had caused divergence of canine and first premolar roots without any resorption. This case report details a diagnosis of two distinct disease processes of different cellular origin namely, focal cemento-ossifying dysplasia and adenomatoid odontogenic tumor in a previously unreported concomitant and contiguous relationship. The diagnosis was determined by a combination of clinical, radiographic, histopathological and surgical evidence. This case highlights two points, first the need to examine all mixed radiolucent-radiopaque lesions with advanced imaging techniques to assess the number and extent of the lesions prior to treatment planning. Second a likely role of periodontal ligament as the tissue source for odontogenic epithelial cells and mesenchymal stem cells required for the development of odontogenic tumors and cemento-osseous dysplasias.
\end{abstract}

Keywords: Adenomatoid odontogenic tumor, cemento-osseous dysplasia, cone beam-CT, extrafollicular, focal cementoosseous dysplasia, odontogenic tumor, periodontal ligament.

\section{INTRODUCTION}

The field of radiology has made considerable progress in recent times. Nonetheless, orthopantomograms (OPG) and occlusal radiographs remain the most widely used imaging modalities for dental \& maxillofacial pathologies. The techniques however have well-known limitations in the diagnosis of lesions that have varying lytic and formative stages. The diagnosis of these lesions needs a combination of clinical history, radiographs, histopathology and often operative findings.

Provisional diagnosis of bony hard lesion of the jaws includes a wide variety of inflammatory, reactive, developmental, endocrine, metabolic and neoplastic entities. This list is significantly reduced by the clinical finding of a slowgrowing, asymptomatic bony hard lesion in close relation to vital teeth in the anterior maxilla in an otherwise healthy young individual. Additional radiographic finding of a welldemarcated mixed radiolucent radiopaque lesion causing divergence of neighboring teeth narrows the diagnosis further. The most likely clinical diagnosis with the mentioned features include central ossifying fibroma (COF), focal cemento-ossifying dysplasia (FCOD) [1], adenomatoid odontogenic tumor (AOT), calcifying odontogenic cyst (COC) and calcifying epithelial odontogenic tumor (CEOT) [2]. When small, these lesions are routinely treated by excisional biopsy that enucleates the lesion and provides tissue for histological diagnosis. This form of management is based

\footnotetext{
*Address correspondence to this author at the Director - OMFP center, 10/B Shree Krishna Mills Compound, Vengurla Road, Hindalga Belgaum 591108, Karnataka, India; Tel: 831 2449951;

E-mails: mandana@darafsh.net, mandanajd@gmail.com
}

on the assumption of a single pathological process the features of which can be adequately discerned on radiological examination. This protocol does not take into account, thus far, unreported cases of two or more contiguous lesions with similar radiographic presentation.

The complex embryonic derivation of the maxillofacial structures causes a widerange of pathologies, particularly in the tooth-bearing areas of the jaws. The development of simultaneous lesions of differing cellular origin, has so far, not been reported in the English literature. Here we present an extra follicular adenomatoid odontogenic tumor (AOT) with calcifying epithelial odontogenic tumor (CEOT) like areas and a concomitant and contiguous focal cemento-osseous dysplasia (FCOD).

\section{CASE REPORT}

A 24-year-old man was referred to the Department of Oral \& Maxillofacial Pathology for evaluation of an asymptomatic bony hard expansion in relation to the buccal aspect of maxillary right canine \& first premolar. Patient's medical history was non-contributory. The lesion was slow growing with one-year duration. It had increased in size slowly for nine months and remained static thereafter. Clinical examination did not show any endodontic or coronal pathologic condition of the neighboring maxillary canine, first premolar, and the maxillary incisors.

Panoramic (Fig. 1) and occlusal radiographs (Fig. 2) showed an oval well-defined unilocular expansile radiolucency measuring $2 \times 1.7 \mathrm{~cm}$. The lesion had a target-like appearance with a central area of floccular calcification and caused divergence of the roots of the right canine and maxil- 
lary first premolar with no root resorption. Based on the clinical and radiographic findings a provisional differential diagnosis was reached. Central ossifying fibroma, focal cemento-ossifying dysplasia and adenomatoid odontogenic tumor were the pathologies considered.

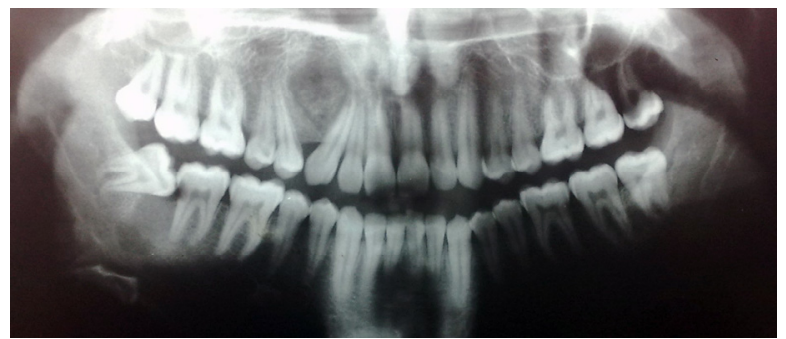

Fig. (1). A panoramic view of the jaws shows a well-defined unilocular radiolucency with central foci of calcification displacing the roots of the maxillary canine and premolar.

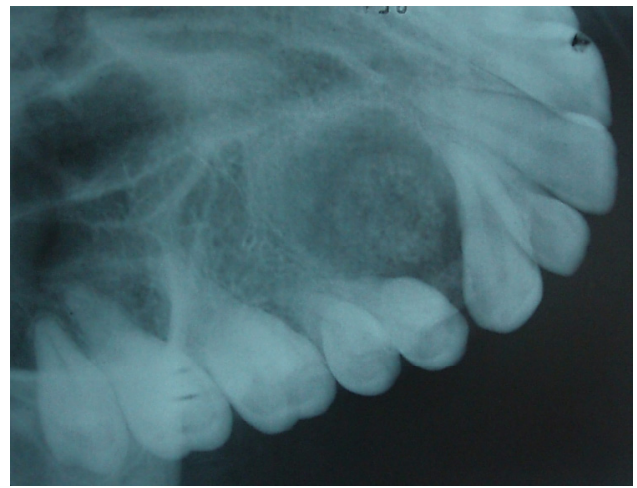

Fig. (2). Occlusal radiograph showing target like lesion between the roots of the canine and first premolar.

Since the lesion was small and appeared well demarcated on radiographs patient was advised to undergo an excisional biopsy. Upon patient's acceptance and medical clearance, the procedure was performed. The lesion was easily separated from the surrounding tissue and enucleated in toto. Gross examination showed an encapsulated lesion with a thick wall, to which multiple irregular gritty pieces of tissue were attached. The overall size of the excised lesion was $3.4 \mathrm{x} 3 \mathrm{x}$ $1.3 \mathrm{~cm}$. The cut- section of the encapsulated lesion showed an intracapsular solid mass with multiple cystic spaces. The overall consistency of the contents was gritty. The entire tissue was prepared for microscopic examination. The tissue blocks were processed for routine paraffin embedding without decalcification.

A whole slide digital scan showed the presence of two distinct and yet closely associated contiguous lesions. A larger well-encapsulated lesion with a thick fibrous wall containing multinodular cellular proliferation with scanty connective tissue and multiple empty areas and, small fibrocellular proliferations interspersed with irregular mineralization partly attached to the extra-capsular surface (Fig. 3A \& B).

The cellular mass within the encapsulated lesion consisted of multiple nodules of varying sizes. The majority of the nodules consisted of cuboidal or columnar epithelial cells that formed nests, rosette-like patterns and duct- like spaces. Tumor droplets (eosinophilic amorphous material) were seen between the cells and in the rosette- like structures. The spaces between the nodules were occupied by interlacing strands of oval, angular and elongated epithelial cells. Basaloid epithelial cells formed single and double layered trabecular or cribriform configurations between and connecting cell rich nodules (Fig. 4A \& B). Few areas showed collections of eosinophilic polyhedral squamous like epithelial cells with well-defined cell borders (Fig. 5A) and intercellular bridges (Fig. 5B). Calcified bodies were found throughout the lesion but were mostly associated with the polygonal cells (Fig. 5B). The mineralization was in the form of globular mineralization that was nearly translucent and, concentric rings (Liesegang pattern). Islands of amyloid- like material that were Congo-red positive and produced characteristic apple green birefringence on polarized microscopy were also found (Fig. 6A-C). The stromal connective tissue was overall scant and loose. The endothelial lining of the blood vessels showed degenerative changes (Fig. 4A).

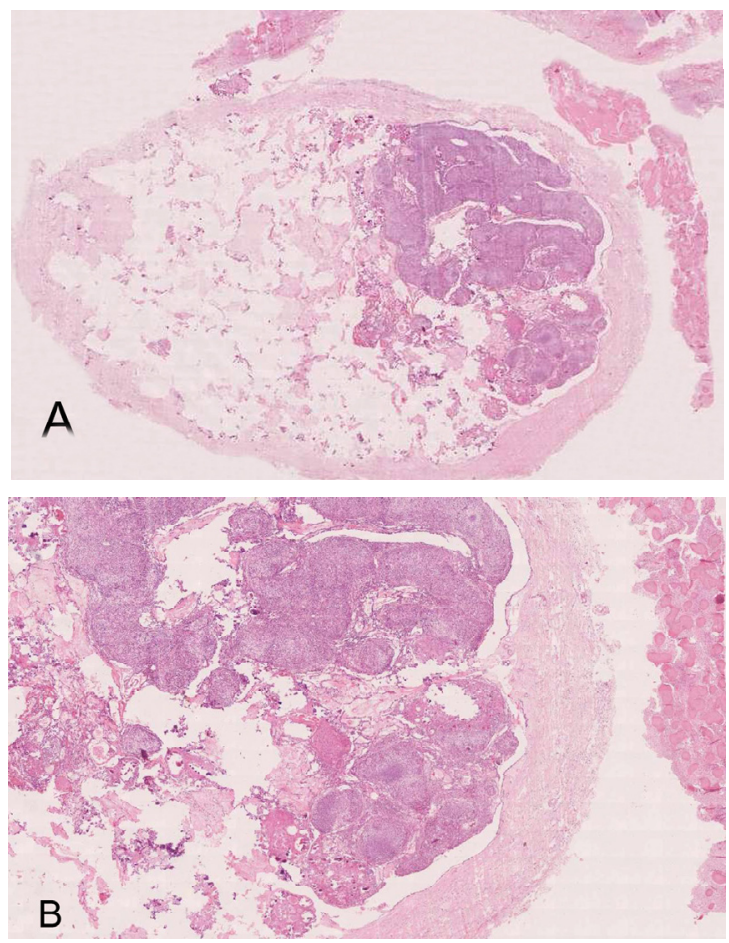

Fig. (3). (A). Thick walled encapsulated lesion with intraluminal epithelial proliferation and extracapsular fibrous tissue with irregular mineralization (H\&E- whole slide digital scan) (B). Multinodular intraluminal solid and cribriform proliferation, CEOT-like areas and extracapsular fibrous lesion with irregular mineralization (H\&E x 40).

The extra capsular lesion consisted of multiple small fragments of fibrocellular mesenchymal tissue interspersed with numerous irregular and interconnecting bone and cementum- like material. An artefactual split existed between parts of the lesion and the fibrous capsule. The fibrocellular collections showed few hemorrhagic areas, thick curvilinear ginger root-like trabeculae with few lacunae and ovoid cementicles with prominent resting lines. Cellular areas showed spindle-shaped fibroblasts. (Fig. 7A \& B). The maturing areas of the lesion showed a confluence of woven and lamellar bone (Fig. 8A \& B). 

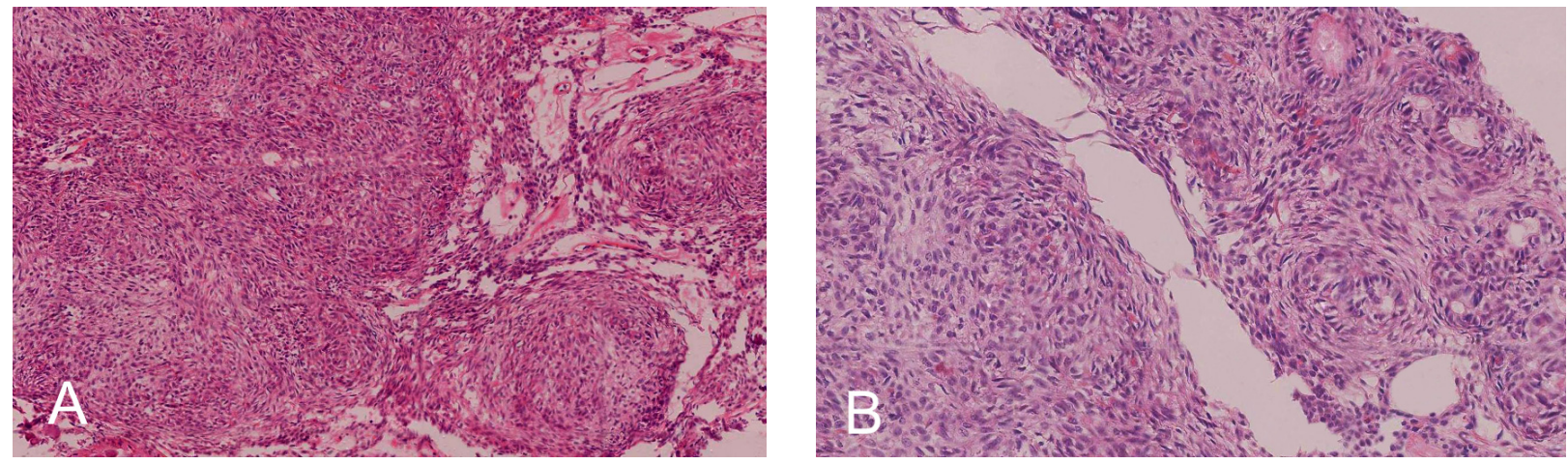

Fig. (4). (A). Intraluminal nodules consisting of cuboidal or columnar epithelial cells in the shape of nests, rosette-like patterns and duct- like spaces with interlacing strands of oval, angular and elongated epithelial cells between nodules.Double layered basaloid epithelial cells in trabecular or cribriform configurations between and connecting cell rich nodules. (H \& E x 200) (B). Tumor droplets (Eosinophilic amorphous material) between the cells and in Rosette-like structures (H\&E x 400).
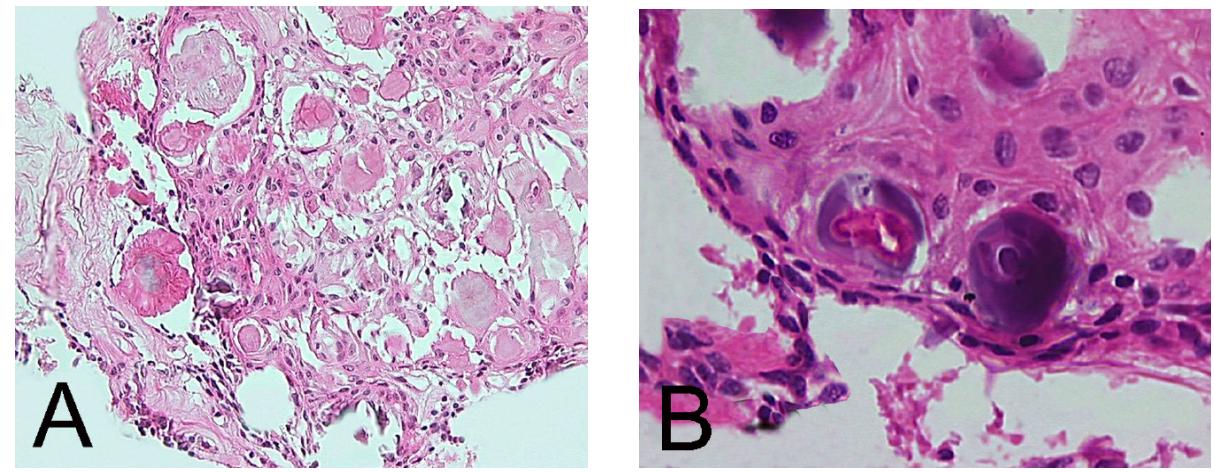

Fig. (5). CEOT -like area. (A). Island of polygonal epithelial cells with intracytoplasmic homogenous material and calcification (H\& $\mathrm{E} x$ 400). (B). Polyhedral epithelial cells exhibiting intercellular bridges and calcification (H\&E x 600).
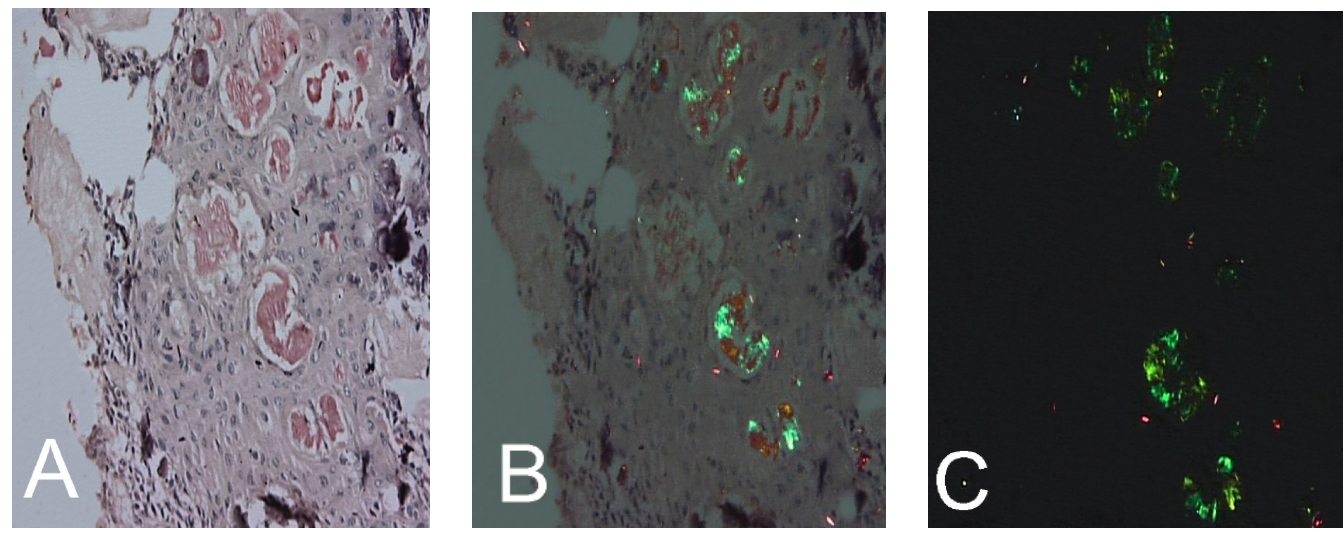

Fig. (6). Congo-red positive islands of amorphous eosinophilic (amyloid like) material (x 200). (A). Light microscopy, (B). Apple green birefringence - partially crossed polarz, (C). Crossed polarz (x200).

The resultant diagnosis was two- fold and reported the existence of two concomitant and contiguous lesions. First lesion was diagnosed as extra-follicular AOT with CEOTlike areas based on clinicopathological findings. The second lesion was diagnosed as an FCOD attached to the fibrous wall of the AOT in an extra capsular relation based on the radiological and histological findings that were confirmed by gross findings of the surgical specimen.

Follow-up was uneventful and showed normal bone healing and the canine and first premolar, were vital two years postoperative.

\section{DISCUSSION}

The clinical presentation of this case suggested the presence of a slow growing lesion in the absence of endodontic involvement of neighboring teeth. Radiological findings showed the lesion to be a well- defined radiolucency with central flecks of calcification in the tooth-bearing anterior region of the maxilla that had displaced the roots of neighboring teeth without causing any resorption. These findings pointed to a non- destructive, non-inflammatory slow growing lesion with an ability to cause some mineralization and led to the clinico-radiographic diagnosis of COF, FCOD, and 

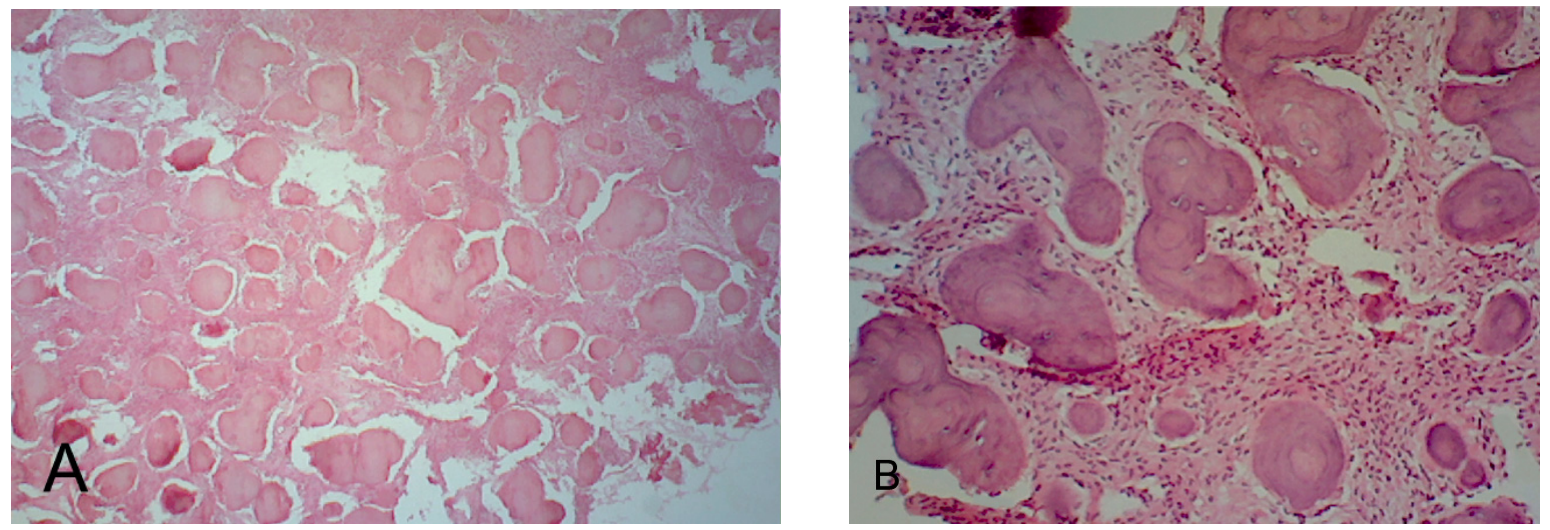

Fig. (7). (A). Fibrocellular tissue interspersed with irregular trabeculae of bone and cementum-like tissue and foci of hemorrhage (H\&E x 40). (B). Fibrocellular connective tissue interspersed with ginger root-like trabeculae showing few lacunae and ovoid cementicles with prominent resting lines (H\& E x 100).
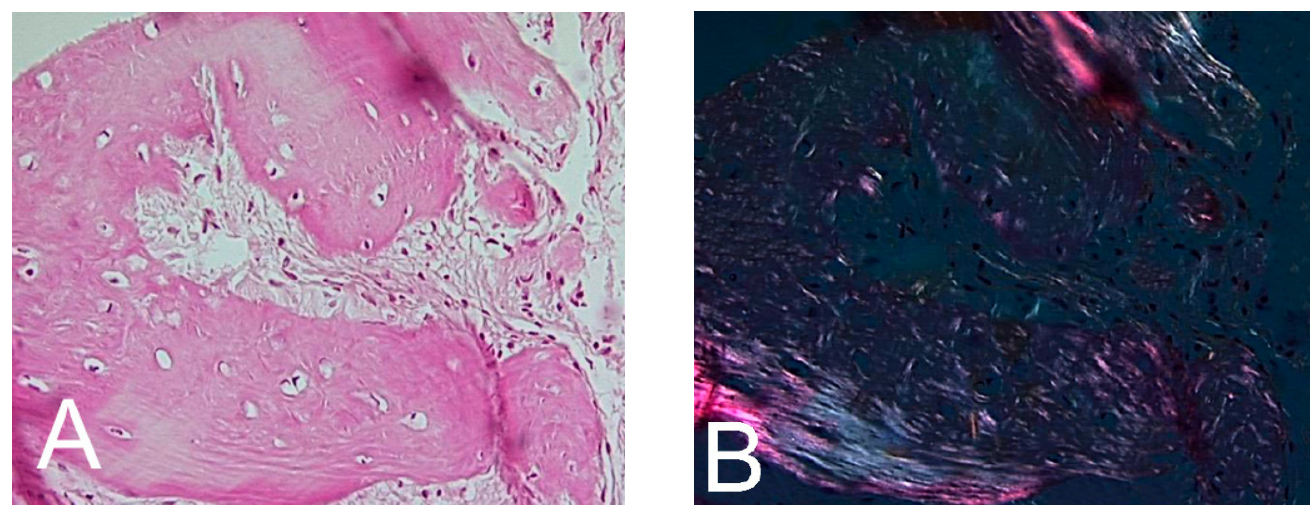

Fig. (8). Confluence of woven and lamellar bone in maturing trabecule, (A). H\&E x 200. (B). Polarized light microscopy x 200.

extrafollicular AOT. The decision to perform an excisional biopsy was routine considering the limited size of the lesion and the differential clinical diagnosis.

The surgical excision unmasked the presence of concomitant and contiguous AOT and FCOD. The microscopic diagnosis of AOT is straightforward especially in cases where the entire lesion is available for examination. The differential diagnosis of fibro-osseous lesions such as FCOD, on the other hand, need to be based on a combination of clinical, radiographic, pathological and surgical findings.

This case fulfilled the clinical and radiographic diagnostic criteria for AOT as well as the microscopic features. The male: female ratio of AOT is $1: 1.9$, which means roughly one third of the patients are males as in the present case. Majority of cases occur in the second and third decades, [3] the age range that included this 24 -year-old patient. The lesion in this patient was non- aggressive [4] marked by a well -defined radiolucency that had caused the displacement of neighboring roots without resorption. It was a unilocular radiolucency located between roots of erupted permanent canine and premolar the most commonly involved [3] teeth with no association with any un erupted teeth [5]. The lesion, therefore, had the typical clinico-radiographic presentation of an extra-follicular AOT, this variety while less common than the follicular type is well-documented accounting for $25 \%$ of the cases [4]. The central flecks of mineralization seen in the radiograph are relatively common and are seen in approximately two-thirds of the extra-follicular variants $[5,6]$. AOT has a remarkably consistent histological presentation. This lesion presented with the characteristic low power presentation of varying sized solid nodules of cuboidal or columnar cells forming nests or rosette-like structures with minimal stromal connective tissue. Additional microscopic features of AOT which include eosinophilic amorphous material or tumor droplets between the cells, [7] duct- like or tubular structures, and nodules that have CEOT- like presentation were also found in this case. The CEOT like areas, while, infrequent [2] are considered to be a normal feature in the continuous histomorphological spectrum of AOT [8]. Varying amounts of calcified material that may appear as irregular calcified bodies, sometimes showing a concentric or Liesegang pattern and globular calcifications in the squamoid or CEOT-like islands are often seen in AOT and were present in this case also. The tumor may contain pools of amyloid [9] that can be demonstrated by the use of Congo red stain and the characteristic apple-green birefringence under polarized light [7]. The presence of amyloid in this case was confirmed accordingly.

Focal cemento-osseous dysplasia is a disease process that affects the tooth-bearing regions of the jaws. FCOD characteristically causes a replacement of normal bone by a mixture of fibrous and osseous components and is thus one of the benign fibro-osseous lesions (BFOL) of the maxillofacial region. BFOLs have overlapping microscopic features and require a combination of clinical, radiographic, microscopic and surgical features for diagnosis. FCOD is the most 
common fibro-osseous lesion of the jaws [10-14] with limited growth potential. Patient follow-up without complete surgical removal is considered adequate treatment after diagnosis of FCOD. Thus, it has to be differentiated from the other BFOLs which include true neoplasms and inflammatory processes with very different treatment requirements. The diagnostic criteria for the diagnosis of FCOD has been outlined by Summerlin DJ and Tomich CE as a set of features that include "(1)a histologic pattern consisting of cellular mesenchymal tissue with intermixed calcifications; (2) radiographic features of a focal radiolucent and/or radiopaque area in the jaws; (3) surgical findings of an easily fragmented hemorrhagic mass of gritty tissue that was removed from the bone with difficulty; and (4) the gross observations of multiple hemorrhagic fragments of variable consistency" [14].

The required histologic pattern of multiple small fragments of fibrocellular mesenchymal tissue interspersed with numerous irregular and interconnecting bone and cementumlike material was seen in this case. Radiographs showed a well- defined radiolucency lesion with central radiopacities. While the presence of a larger concurrent and contiguous disease processes with similar radiographic features could have affected the presentation the possible contribution of the FCOD to the overall picture cannot be ruled out. Typically surgical excision of FCOD demonstrates the close association of this lesion to the bone. The tenuous fibrous attachment between the lesion and the bone makes it difficult to separate the lesional tissue from the bone. A similar situation was found in this case however the fibrous attachment was between the FCOD and the capsule of the AOT. Gross showed multiple irregular gritty pieces of tissue that remained attached to the extra capsular surface of the AOT. Based on these combined criteria the diagnosis of FCOD was established.

The combination of radiographic and surgical features observed in this case ruled out other BFOLs including COF the greatest diagnostic challenge for FCOD. The pathologic differentiation of FCOD and COF based on histopathology alone is not often possible due to the extensive overlap of microscopic features presented by the two entities. However, the use of certain features that have different presentations can help in histological diagnosis of the lesions. [14]. The characteristic curvilinear thick interconnected trabeculae seen in FCOD are present in COF occasionally, at the periphery of the lesion. While, in FCOD they are dispersed throughout the lesion as in the current case. Free hemorrhage is seen in $40 \%$ of COFs. Whereas, it is present in the lesion borders in COF, in FCOD it is found within the lesion. Free hemorrhage becomes progressively infrequent with progressive mineralization or maturation of FCOD lesions [15].

AOT is an uncommon epithelial odontogenic tumor that represents approximately $3 \%$ of all odontogenic tumors. The combination of an extra follicular variant with CEOT -like areas in a male patient makes this case an uncommon presentation of AOT.

FCODs are most commonly seen in women in the fourth decade of life affecting the posterior mandible. Only $11 \%$ of FCODs are found in males, and less than $16 \%$ occur in individuals in the third decade of life [14]. The rate of occur- rence of FCOD in the maxilla is limited to $11 \%$ of the cases. The lesions are also mostly confined to a periapical location or edentulous spaces at the site of previous extractions. The present lesion, however, was not at either of these typical sites being present between the teeth with no association to the periapical region of the teeth or a previous extraction site. Considering the demographics and location of occurrence this case is a relatively rare presentation of FCOD.

Various odontogenic cysts and tumors [16], such as dentigerous cyst [7], Calcifying epithelial odontogenic cyst [17], ameloblastoma [18], and odontomas have been reported in association with AOT. However, these associations have been limited to foci resembling one of the lesions in AOT and vice versa. FCOD rarely shows cystic changes and, some instances of coexisting inflammatory conditions of the bone have been reported [19]. There are, however, no reported cases of a concurrent and contiguous odontogenic tumor with a fibro-osseous lesion. Thus, the AOT and FCOD presented here are the first occurrence of a fibro-osseous and odontogenic tumor that while attached to each other were independent lesions preserving their characteristic features.

This close association of two entirely different pathological processes is a manifestation of the complex biological systems involved in the development of the maxillofacial and dental structures that place tissues with broad functionality and characteristics in proximity.

AOT is an epithelial odontogenic tumor widely believed to arise from the epithelial remnants [20]. The source for the epithelial remnants may be either dental lamina (Serres pearls) or Hertwigs epithelial root sheet (cell rests of Malassez) [21]. Philipsen \& Reichart consider Serres pearls distributed along the gubernaculum dentis as the most likely source capable of producing the central and peripheral variants of AOT [20]. The role of cell rests of Mallassez as the epithelial origin is supported by the reported case of AOT arising in the periodontal ligament (PDL) [22].

The pathogenesis of FCOD is not clear, however, the proximity of FCOD with tooth apices and the frequent presence of cementum like mineralization in the entity [19] have led to the common acceptance of fibroblastic mesenchymal stem cells in the PDL as the origin of the disease process $[11,14,17]$. Other views suggest defects in the medullary bone, with faults in the extra-ligamentary bone remodeling triggered by local factors and possibly influenced by hormonal imbalance or a combination of the periodontal and bone origin [19, 23].

The presentation of FCOD and AOT in this case can unify the source tissue for these lesions or, at-least, some variants of each disease. Periodontal ligament is the only tissue that can simultaneously provide the mesenchymal stem cells responsible for the development of FCOD and the odontogenic epithelium in the form of cell rests of Malassez required for the development of AOT. These findings need to be further examined using molecular, and genetic tests.

The overlapping radiographic features of the AOT and FCOD, in this case, while previously unreported and exceedingly rare highlights the need for more exhaustive radiographic examination. While the use of basic radiographic technique buccal object rule applied to IOPA, and occlusal 
views can help localize and separate superimposed lesions. A greater and more uniform success in visualizing the details and multiplicity of such lesions is achieved by use of conventional CT or Cone-beam CT [24]. These radiographic techniques should be used to examine lesions such as FCOD where a decision to defer treatment is standard protocol.

\section{CONFLICT OF INTEREST}

The authors confirm that this article content has no conflict of interest.

\section{ACKNOWLEDGEMENTS}

Declared none.

\section{REFERENCES}

[1] Eversole L. Clinical outline of oral pathology. Shelton, Conn.: House-USA. People's Medical Pub 2011.

[2] de Matos F, Nonaka C, Pinto L, de Souza L, de Almeida Freitas R. Adenomatoid odontogenic tumor: retrospective study of 15 cases with emphasis on histopathologic features. Head Neck Pathol 2012; 6(4): 430-7.

[3] Philipsen H, Reichart P, Siar C, et al. An updated clinical and epidemiological profile of the adenomatoid odontogenic tumour: a collaborative retrospective study. J Oral Pathol Med 2007; 36(7): 383-93.

[4] Philipsen H, Reichart P. Adenomatoid odontogenic tumour: facts and figures. Oral Oncol 1999; 35(2): 125-31.

[5] Philipsen H, Reichart P, Zhang K, Nikai H, Yu Q. Adenomatoid odontogenic tumor: biologic profile based on 499 cases. J Oral Pathol Med 1991; 20(4): 149-58.

[6] Philipsen PH, Reichart AP, Nikai H. The adenomatoid odontogenic tumour (AOT): an update. Oral Med Pathol 1997; 2(2): 55-60.

[7] Leon J, Mata G, Fregnani E, et al. Clinicopathological and immunohistochemical study of 39 cases of adenomatoid odontogenic tumour: a multicentric study. Oral Oncol 2005; 41(8): 83542.

[8] Mosqueda-Taylor A, Carlos-Bregni R, Ledesma-Montes C, Fillipi $\mathrm{R}$, de Almeida O, Vargas P. Calcifying epithelial odontogenic tumor-like areas are common findings in adenomatoid odontogenic tumors and not a specific entity. Oral Oncol 2005; 41(2): 214-5.

[9] Takeda Y, Kudo K. Adenomatoid odontogenic tumor associated with calcifying epithelial odontogenic tumor. Int J Oral Maxillofac Sur 1986; 15(4): 469-73.

[10] Slootweg P. Maxillofacial fibro-osseous lesions:classification and differential diagnosis. Semin Diagn Pathol 1996; 13: 104-12.

[11] Waldron C. Fibro-osseous lesions of the jaws. J Oral Maxillofac Sur 1985; 43(4): 249-62.

[12] Waldron C. Fibro-osseous lesions of the jaws. J Oral Maxillofac Sur 1993; 51(8): 828-35.

[13] Kramer I, Pindborg J, Shear M. Histological typing of odontogenic tumours. Berlin: Springer-Verlag 1992.

[14] Summerlin D, Tomich C. Focal cemento-osseous dysplasia: A clinicopathologic study of 221 cases. Oral Sur Oral Med Oral Pathol 1994; 78(5): 611-20.

[15] Su L, Weathers D, Waldron C. Distinguishing features of focal cemento-osseous dysplasias and cemento-ossifying fibromas: I.A pathologic spectrum of 316 cases. Oral Sur Oral Med Oral Pathol Oral Radiol Endodontol 1997; 84(3): 301-9.

[16] Rick G. Adenomatoid odontogenic tumor. Oral Maxillofac Sur Clin North Am 2004; 16(3): 333-54.

[17] Barnes L. Pathology and genetics of head and neck tumours. Lyon IARC Press 2005.

[18] Raubenheimer E, Heerden W, Noffke C. Infrequent clinicopathological findings in 108 ameloblastomas. J Oral Pathol Med 1995; 24(5): 227-32.

[19] Kawai T, Hiranuma H, Kishino M, Jikko A, Sakuda M. Cementoosseous dysplasia of the jaws in 54 Japanese patients. Oral Sur Oral Med Oral Pathol Oral Radiol Endodontol 1999; 87(1): 107-14.

[20] Philipsen H, Samman N, Ormiston I, Wu P, Reichart P. Variants of the adenomatoid odontogenic tumor with a note on tumor origin. $J$ Oral Pathol Med 1992; 21(8): 348-52.

[21] Philipsen H, Reichart P. The development and fate of epithelial residues after completion of the human odontogenesis with special reference to the origins of epithelial odontogenic neoplasms, hamartomas and cysts. Oral Biosci Med 2004; 1(3): 171-9.

[22] Jham B, Passos J, Carmo M, Gomes C, Mesquita R. Adenomatoid odontogenic tumor originated in the periodontal ligament. Oral Oncol Extra 2006; 42(7): 268-71.

[23] Neville B. Oral and maxillofacial pathology. St. Louis, Mo.: Saunders/Elsevier 2009.

[24] Quereshy F, Savell T, Palomo J. Applications of Cone Beam Computed Tomography in the Practice of Oral and Maxillofacial Surgery. J Oral Maxillofac Sur 2008; 66(4): 791-6.

Received: December 20, 2014

Revised: March 19, 2015

Accepted: May 25, 2015

(C) Rezvani et al.; Licensee Bentham Open.

This is an open access article licensed under the terms of the Creative Commons Attribution Non-Commercial License (http://creativecommons.org/licenses/by-nc/3.0/) which permits unrestricted, non-commercial use, distribution and reproduction in any medium, provided the work is properly cited. 